\title{
Insecticidal, Antimalarial, and Antileishmanial Effects of Royal Jelly and Its Three Main Fatty Acids, trans-10-Hydroxy-2-decenoic Acid, 10-Hydroxydecanoic Acid, and Sebacic Acid
}

\author{
Abeer Mousa Alkhaibari (iD) ${ }^{1}$ and Abdullah D. Alanazi $\mathbb{D i D}^{2}$ \\ ${ }^{1}$ Department of Biology, Faculty of Science, University of Tabuk, Tabuk 71491, Saudi Arabia \\ ${ }^{2}$ Department of Biological Science, Faculty of Science and Humanities, Shaqra University, Ad-Dawadimi 11911, Saudi Arabia \\ Correspondence should be addressed to Abeer Mousa Alkhaibari; aalkhaibari@ut.edu.sa
}

Received 23 November 2021; Revised 31 December 2021; Accepted 4 January 2022; Published 20 January 2022

Academic Editor: Sekar Vijayakumar

Copyright (c) 2022 Abeer Mousa Alkhaibari and Abdullah D. Alanazi. This is an open access article distributed under the Creative Commons Attribution License, which permits unrestricted use, distribution, and reproduction in any medium, provided the original work is properly cited.

\begin{abstract}
Natural products and their derivatives as an inexpensive, accessible, and useful alternative medicine are broadly applied for the treatment of a wide range of diseases and infectious ones. The present study was designed to evaluate the insecticidal, antimalarial, antileishmanial, and cytotoxic effects of royal jelly and its three main fatty acids (trans-10-hydroxy-2-decenoic acid (10-H2DA), 10-hydroxydecanoic acid (10-HDAA), sebacic acid (1,10-decanedioic acid)). Insecticidal activity of RJ and 10-H2DA, 10-HDAA, and sebacic acid was performed against healthy 4 th instar larvae at $25 \pm 2^{\circ} \mathrm{C}$. Antiplasmodial and antileishmanial effects of RJ and 10-H2DA, 10-HDAA, and sebacic acid were also performed against chloroquine-resistant Plasmodium falciparum K1-strain and Leishmania major amastigotes according to the Malstat method and macrophage model, respectively. In addition, the level of nitric oxide (NO) production in J774-A1 macrophages cells, plasma membrane permeability, and caspase-3-like activity and cytotoxicity effects of RJ and 10-H2DA, 10-HDAA, and sebacic acid against human embryonic kidney 293 (HEK239T cells) were evaluated. Considering the insecticidal activity, the results showed that the lethal concentration $50 \%$ value for RJ, 10-H2DA, 10HDAA, and sebacic acid was $24.6,31.4,37.8$, and $44.7 \mu \mathrm{g} / \mathrm{mL} \mu \mathrm{g} / \mathrm{mL}$, respectively. RJ, 10-H2DA, 10-HDAA, and sebacic acid showed potent $(P<0.0001)$ antileishmanial effects with $\mathrm{IC}_{50}$ values ranging from 2.4 to $8.4 \mu \mathrm{g} / \mathrm{mL}$. Various concentrations of $\mathrm{RJ}$, 10-H2DA, 10-HDAA, and sebacic acid significantly $(P<0.05)$ increased the production of NO, plasma membrane permeability, and caspase-3-like activity level as a dose-dependent response. Considering the cytotoxicity, SIs $>10$ of these compounds exhibited their specificity to parasites and safety against human HEK239T normal cells. The results of the present investigation revealed the promising insecticidal, antimalarial, and antileishmanial effects of RJ and its three main fatty acids (10-H2DA, 10HDAA, and sebacic acid). However, more studies are required to confirm the mechanisms of action mode of these compounds as well as their efficacy in animal models and clinical settings.
\end{abstract}

\section{Introduction}

According to the World Health Organization (WHO) reports, vector-borne diseases account for nearly $17 \%$ of all infectious diseases, with a mortality rate of 700,000 deaths each year [1]. Aedes aegypti is considered as the principal vector recognized to transmit a number of important vector-borne infections such as malaria, dengue, chikungunya, and Zika [2,3]. Today, one of the most important approaches for controlling insects is the use of insecticides (e.g., diflubenzuron, alpha-cypermethrin, malathion, and deltamethrin) $[4,5]$. The current studies demonstrated that the too much and persistent use of chemical and synthetic insecticides has reduced their efficacy and raised various ecological concerns such as emerging of drug resistance to insecticides, ecological imbalance, and outcomes to animals [6, 7]. Accordingly, these problems and limitations have led researchers to pay more attention to finding novel insecticides with high efficacy and eco-friendly properties around the world. 
Malaria is a serious vector-borne infection, which causes about 430,000 deaths yearly, generally in African children [8]. Among the Plasmodium species in human, Plasmodium vivax and $P$. falciparum are well known as the most common and deadly Plasmodium species, respectively [9, 10]. In recent decades, there have been numerous reports on side effects as well as drug resistance of Plasmodium spp. (mainly $P$. falciparum malaria) to current antimalarial drugs [11]. So finding and introducing a new antimalarial drug especially from natural resources with the lower toxicity and the highest performance is necessary for malaria treatment.

Leishmaniasis as a neglected tropical infection is caused by the parasitic species Leishmania, which infects about 12 million people each year in 98 countries worldwide [12]. The disease is observed in humans in different forms, including cutaneous leishmaniasis, cutaneous-mucosal leishmaniasis, and visceral leishmaniasis [13]. In chemotherapy as the most important method of treating leishmaniasis, various drugs such as meglumine antimoniate and Pentostam are used; however, the previous studies have reported that the majority of the synthetic antileishmanial drugs have been associated with some disadvantages and limitations such as emerging drug resistance and adverse side effects [14, 15].

From a long time ago, natural products have been well known as a valuable and effective resource for treating a broad spectrum of diseases, including cardiovascular, cancers, and neurological [16]. Among the natural products, beehive derivatives and products including royal jelly, honey, and propolis are considered as one of the best healthpromoting products [17]. Royal jelly (RJ) is a gelatinous material secreted from the apical glands of young nurse honey bees with various pharmacological and biological benefits such as anticancer, antioxidant, anti-inflammatory, and antimicrobial [18-20]. Considering the compounds in RJ, previous studies have demonstrated that the main composition of RJ is water (50-60\%), proteins (18\%), carbohydrates (7-18\%), and lipids (3-8\%), respectively [21]. The lipid composition of RJ consists of $80-85 \%$ fatty acids, composed of proteins that are attributed to its biological properties [22]. Reviews showed that the major fatty acids present in RJ are (i) trans-10-hydroxy-2-decenoic acid (10H2DA), an unsaturated hydroxyl fatty acid which consists of $>50 \%$ of the free FAs; (ii) 10-hydroxydecanoic acid (10HDAA), as a saturated hydroxyl fatty acid, 10-HDAA; (iii) sebacic acid (SEA, 1,10-decanedioic acid), a dicarboxylic fatty acid, comprising 3.3\% of the total acids in RJ $[23,24]$.

Based on what has been said about the biological and pharmacological activities of RJ and also the limitations, problems, and side effects of the current insecticidal, antileishmanial, and antimalarial agents, in this study, we decided to assess the insecticidal, antiplasmodial, antileishmanial, and cytotoxic effects of RJ and its three main fatty acids (10-H2DA, 10-HDAA, and sebacic acid).

\section{Materials and Methods}

2.1. Royal Jelly. Royal jelly materials were obtained from Langstroth hives containing colonies of the A. mellifera grown in Tabuk, Saudi Arabia, from June 2021. Samples were dissolved with normal saline and filtered under a vacuum by means of filter paper (Whatman membrane, England). Samples $(50 \mathrm{mg}$ ) were diluted in distilled water and then ultrasonicated for $1 \mathrm{~h}$. In the next step, the sample solution was centrifuged at $6000 \mathrm{rpm}$ for 10 minutes, whereas the upper phase was applied as the sample solution and was stored at $-20^{\circ} \mathrm{C}$ until testing.

\subsection{Secondary Metabolites Contents}

2.2.1. Total Phenol Content. The total phenol content in the RJ sample was assessed based on the technique explained by Singleton et al. [25]. To do this, $50 \mu \mathrm{L}$ of RJ solution ( $50 \mathrm{mg} /$ $\mathrm{mL}$ ) was added to $250 \mu \mathrm{L}$ of Folin-Ciocalteu's reagent $(0.2 \mathrm{~N})$ for $6 \mathrm{~min}$, and then $0.2 \mathrm{~mL}$ of sodium carbonate $(7.5 \%)$ was put into the tested tubes. After $120 \mathrm{~min}$ incubation at room temperature, the absorbance of suspension was determined at $760 \mathrm{~nm}$. Distilled water was used as the blank solution. Total phenol content was reported as $\mathrm{mg}$ gallic acid equivalents (GAE)/g.

2.2.2. Total Flavonoid Content. The determination of flavonoid content was performed according to the method explained by El-Guendouz et al. In this way, $200 \mu \mathrm{L}$ of RJ solution was poured into the tubes with $200 \mu \mathrm{L}$ aluminum chloride $(20 \%)$. The tubes were then incubated at room temperature for $60 \mathrm{~min}$, and their absorbance was determined at $420 \mathrm{~nm}$ [26]. The total flavonoid content was reported as milligram of quercetin equivalents per gram of RJ (mg QE/g RJ).

2.2.3. Total Protein Content. Bradford technique was used to determine the protein content. Briefly, the RJ sample (200 mg) was added to a tube containing $10 \mathrm{~mL}$ methanol/water (50/50; $\mathrm{v} / \mathrm{v}$ ); the suspension was sonicated for $1 \mathrm{~h}$. In the next step, the $\mathrm{pH}$ of the suspension was adjusted to 2.5 with phosphoric acid and was then diluted 10 times. Next, $5 \mathrm{~mL}$ of Bio-Rad reagent was mixed with $250 \mu \mathrm{L}$ of RJ solutions, and the absorbance of the mixture was determined at $595 \mathrm{~nm}$. The total protein content was reported as a percentage by means of the bovine serum albumin standard curve [27].

2.3. Chemicals. trans-10-hydroxy-2-decenoic acid, 10hydroxydecanoic acid, sebacic acid (1,10-decanedioic acid), and MTT (3-(4,5-dimethylthiazol-2-yl)-2,5-diphenyltetrazolium bromide) powder in high purity were purchased from Sigma-Aldrich (Germany).

2.4. Insecticidal Activity. Ae. aegypti eggs were provided from the Department of Biology, Faculty of Science, University of Tabuk, Saudi Arabia. The insecticidal effects of RJ, 10-H2DA, 10-HDAA, and sebacic acid were carried out based on the method described by Huong et al. [28]. To do this, various concentrations of RJ, 10-H2DA, 10-HDAA, and sebacic acid $(12.5,25,50$, and $100 \mu \mathrm{g} / \mathrm{mL})$ were put in a $500 \mathrm{~mL}$ beaker, and $150 \mathrm{~mL}$ water was added with 20 healthy 4th instar larvae at $25 \pm 2^{\circ} \mathrm{C}$. Larva mortality was determined after $24 \mathrm{~h}$ of 
incubation; lethal concentration 50\% $\left(\mathrm{LC}_{50}\right)$ was calculated via the Probit test in SPSS software for RJ, 10-H2DA, 10-HDAA, and sebacic acid. All tests were carried out in triplicate, and during experiments, no nutritional complement was added, whereas DMSO was considered as the control group.

2.5. Antiplasmodial Activity. The Malstat method was used to determine the antiplasmodial effects of different concentrations of RJ, 10-H2DA, 10-HDAA, and sebacic acid $(12.5,25,50$, and $100 \mu \mathrm{g} / \mathrm{mL})$ against $P$. falciparum $\mathrm{K} 1$-strain [29]. Parasites were firstly incubated with the human erythrocytes (red blood cells, RBC) in RPMI-1640 medium improved with $10 \%$ human serum at $37^{\circ} \mathrm{C}$ with low oxygen atmosphere $\left(3 \% \mathrm{O}_{2}, 4 \% \mathrm{CO}_{2}\right.$, and $\left.93 \% \mathrm{~N}_{2}\right)$. The infected human RBC $(0.2 \mathrm{~mL}, 1 \%$ parasitaemia, and $2 \%$ hematocrit) were treated with various concentrations of RJ, 10-H2DA, 10-HDAA, and sebacic acid in each well and were incubated for 3 days. Then, tested plates were frozen at $-20^{\circ} \mathrm{C}$. In the next step, in a new plate, $0.1 \mathrm{~mL}$ of Malstat reagent was mixed with $0.02 \mathrm{~mL}$ of suspension of hemolysed parasite and incubated for $15 \mathrm{~min}$ at $21^{\circ} \mathrm{C}$. After this time, $0.02 \mathrm{~mL}$ of NBT/PES solution was added to the plates and was incubated again for $120 \mathrm{~min}$ in the dark. Finally, the absorbance of each well was recorded at $655 \mathrm{~nm}$ with the ELISA reader. The $50 \%$ inhibitory concentrations $\left(\mathrm{IC}_{50}\right)$ were also calculated via the Probit test in SPSS software.

2.6. Antileishmanial Effects. L. major promastigotes (MRHO/IR/75/ER) and murine macrophage cells (J774A1) were cultured at RPMI 1640 complemented with $15 \%$ heat-inactivated fetal calf serum (FCS), streptomycin $(100 \mu \mathrm{g} / \mathrm{mL})$, and penicillin $(200 \mathrm{IU} / \mathrm{mL})$ and at Dulbecco's modified eagle's medium (DMEM) improved with $10 \% \mathrm{FCS}$ at $37^{\circ} \mathrm{C}$ in $5 \% \mathrm{CO}_{2}$. Firstly, J774-A1 cells $\left(5 \times 10^{5}\right.$ cells $/ \mathrm{mL}$ ) were transferred in sterile 6 -cell plates (with 1 $\mathrm{cm}^{2}$ coverslip implanted on their floor) and incubator at $37^{\circ} \mathrm{C}$ for 24 hours with $5 \% \mathrm{CO}_{2}$ to adhere to macrophages. After 24 hours, the plates were removed from the incubator and washed with sterile warm saline phosphate buffer. Then, $1 \mathrm{~mL}$ of $L$. major promastigotes $\left(5 \times 10^{6}\right)$ in the stationary phase was added to plates and kept warm at $37^{\circ} \mathrm{C}$ for 4 hours; then, the wells were washed with RPMI1640 medium to remove free promastigotes. In the next step, $1 \mathrm{~mL}$ of RPMI1640 medium containing different concentrations of RJ, 10-H2DA, 10-HDAA, and sebacic acid $(1,2,4$, and $8 \mu \mathrm{g} / \mathrm{mL})$ and MA was added to the wells and was incubated for 48 hours. The slides were then fixed with methanol and stained with Gamisa dye diluted with water in a ratio of $1: 10$. The results were estimated by calculating the mean number of amastigotes inside 100 macrophages. The $\mathrm{IC}_{50}$ values were also calculated via the Probit test in SPSS software. Examinations were carried out in triplicate [30].

2.7. Plasma Membrane Permeability. In order to determine the plasma membrane permeability, L. major $\left(1 \times 10^{6}\right.$ cells $/ \mathrm{mL}$ ) were incubated with different concentrations of
RJ, 10-H2DA, 10-HDAA, and sebacic acid (12.5, 25, 50, and $100 \mu \mathrm{g} / \mathrm{mL}$ ) and then Sytox Green stain was applied based on the kit instructions. Nontreated parasites and those treated with $2.5 \%$ of Triton X-100 (Sigma-Aldrich) were determined as the negative and positive control, respectively. The plasma membrane permeability was calculated by means of a microplate reader (BMG Labtech, Germany) for $4 \mathrm{~h}$ [30].

2.8. Effect on Nitric Oxide (NO) Production. The effect of RJ, 10-H2DA, 10-HDAA, and sebacic acid on the NO release of macrophage cells was performed via Griess reaction for nitrites. In summary, macrophage cells $\left(1 \times 10^{6}\right.$ cells $\left./ \mathrm{mL}\right)$ were exposed with various concentrations of RJ, 10-H2DA, 10-HDAA, and sebacic acid $(1,2$, and $4 \mu \mathrm{g} / \mathrm{mL})$ for $72 \mathrm{~h}$. Then, $0.1 \mathrm{~mL}$ of collected supernatants was transferred into a 96-well microplate, and $60 \mu \mathrm{L}$ of Griess reagents A and B was put within each well. Lastly, the production of NO was determined by reading the plates at $540 \mathrm{~nm}$ in an ELISA reader (BioTek-ELX800) [30].

\subsection{Evaluating the Caspase-3-Like Activity of Extract-Treated} Promastigotes. Caspase-3-like activity of L. major promastigotes treated with RJ, 10-H2DA, 10-HDAA, and sebacic acid was assessed through the colorimetric protease (Sigma, Germany) technique according to the manufacturer guidelines. In this way, the caspase-3-like activity level was measured based on the rate of color spectrophotometric produced through the release of a molecule (pNA attached to the substrate) under the enzyme caspase- 3 activity. Briefly, the promastigotes $\left(10^{6}\right)$ were incubated with RJ, 10-H2DA, 10-HDAA, and sebacic acid for $48 \mathrm{~h}$ and were centrifuged at $700 \mathrm{rpm}$ for 5 minutes at $4^{\circ} \mathrm{C}$. In the next step, the cell residue was lysed, and the cell lysate was centrifuged again at 20,000 rpm for 10 minutes. Lastly, supernatant of reaction $(5 \mu \mathrm{L})$ was added to the $85 \mu \mathrm{L}$ of buffer, and $10 \mu \mathrm{L}$ of caspase 3 (pNADEVD-Ac) solution and the mixture was incubated for $120 \mathrm{~min}$ at $37^{\circ} \mathrm{C}$. The caspase-3-like activity was determined through the light absorption at $405 \mathrm{~nm}$ with the ELISA reader [29].

2.10. Cytotoxicity Effects. The cytotoxic effect of RJ, 10-H2DA, 10-HDAA, and sebacic acid against human embryonic kidney 293 (HEK239T cells) was evaluated using the colorimetric MTT (3-(4,5-dimethylthiazol-2-yl)-2,5-diphenyltetrazolium bromide) assay according to the method described elsewhere [30]. HEK239T cells were cultured in DMEM, supplemented with $10 \%$ fetal bovine serum and streptomycin $(100 \mu \mathrm{g} / \mathrm{mL})$ and penicillin $(200 \mathrm{IU} / \mathrm{mL})$. Next, HEK239T cells $\left(5 \times 10^{4} / \mathrm{mL}\right)$ were treated with RJ, 10-H2DA, 10-HDAA, and sebacic acid (12.5, $25,50,100$, and $200 \mu \mathrm{g} / \mathrm{mL}$ ) for $48 \mathrm{~h}$ in microplates at $37^{\circ} \mathrm{C}$ with $5 \% \mathrm{CO}_{2}$. The $50 \%$ cytotoxic concentrations ( $\mathrm{CC}_{50}$ values) were calculated by means of the Probit test in SPSS software. Selectivity indices $\left(\mathrm{CC}_{50} / \mathrm{IC}_{50}\right)$ were also recorded for each tested drug [27]. 
2.11. Statistical Analysis. The statistical analysis was performed by the SPSS statistical package, version 22.0 (SPSS, Inc.). To compare the results among tested groups, we applied the unpaired samples $t$-test, one-way analysis of variance (ANOVA), and Dunnett's test. $P<0.05$ was measured statistically significant.

\section{Results}

3.1. Secondary Metabolites Contents of RJ. The findings of the secondary metabolites analysis of RJ displayed that total phenolic and flavonoid content were $83.6 \pm 0.31$ (mg GEA/g DW) and $1.78 \pm 0.023(\mathrm{mg} \mathrm{QE} / \mathrm{g} \mathrm{DW})$, respectively; the results also showed that the total protein content of the RJ sample was $11.3 \%$ (Table 1).

3.2. Insecticidal Effects. Table 2 revealed that the larvicidal effects of RJ, 10-H2DA, 10-HDAA, and sebacic acid were remarkable $(P<0.001)$ against $A$ e. aegypti larva. The results showed that among the tested compounds sebacic acid and RJ displayed the lowest and highest larvicidal effects against $A e$. aegypti larva with the $\mathrm{LC}_{50}$ of 44.7 and $24.6 \mu \mathrm{g} / \mathrm{mL}$, respectively.

3.3. Antimalarial Activity. The obtained findings demonstrated that RJ, 10-H2DA, 10-HDAA, and sebacic acid had considerable $(P<0.001)$ antiplasmodial effects against $P$. falciparum. The $\mathrm{IC}_{50}$ value for RJ was $7.62 \mu \mathrm{g} / \mathrm{mL}$, while the $\mathrm{IC}_{50}$ value for $10-\mathrm{H} 2 \mathrm{DA}, 10-\mathrm{HDAA}$, and sebacic acid was $2.41,2.65$, and $3.1 \mu \mathrm{g} / \mathrm{mL}$, respectively (Table 3), indicating that the lowest and highest antimalarial activities were observed in RJ and 10-H2DA, respectively.

3.4. Antileishmanial Effects. Our findings also exhibited that RJ, 10-H2DA, 10-HDAA, and sebacic acid had considerable $(P<0.001)$ antileishmanial effects against intracellular amastigotes of $L$. major. The $\mathrm{IC}_{50}$ value for $\mathrm{RJ}$ was $8.14 \mu \mathrm{g} / \mathrm{mL}$, while the $\mathrm{IC}_{50}$ value for 10-H2DA, 10-HDAA, and sebacic acid was 3.8, 3.77, and $4.13 \mu \mathrm{g} / \mathrm{mL}$, respectively (Table 4 ). These findings indicated that although all tested compounds showed higher antileishmanial effects against intracellular amastigotes of L. major, the lowest and highest antileishmanial activities were observed in RJ and 10-H2DA, respectively.

3.5. Cytotoxicity Effects. Considering the cytotoxicity effects of RJ, 10-H2DA, 10-HDAA, and sebacic acid, the obtained results of the MTT assay demonstrated that $\mathrm{CC}_{50}$ value for RJ, 10-H2DA, 10-HDAA, and sebacic acid was 117.3, 74.4, 81.3 , and $92.6 \mu \mathrm{g} / \mathrm{mL}$; subsequently, their $\mathrm{SI}>10$ of these compound exhibited their specificity to parasites and safety against human HEK239T normal cells (Table 5), indicating that among the tested compounds the lowest and highest cytotoxic effects were observed in RJ and sebacic acid, with the SI value of 13.9 and 22.4, respectively.

3.6. The Effect on the Plasma Membrane Permeability. Here, we evaluated the plasma membrane permeability of the L. major promastigotes treated with RJ, $10-\mathrm{H} 2 \mathrm{DA}, 10-$
HDAA, and sebacic acid. The results of relative fluorescence units revealed that the promastigotes treated with RJ, 10H2DA, 10-HDAA, and sebacic acid as a dose-dependent response changed the permeability of plasma membrane by Sytox Green (Figure 1).

3.7. Evaluating NO Production. As shown in Figure 2, various concentrations of RJ, 10-H2DA, 10-HDAA, and sebacic acid $(1,2$, and $4 \mu \mathrm{g} / \mathrm{mL})$ significantly $(P<0.05)$ increased the production of $\mathrm{NO}$ as a dose-dependent pattern in comparison to the nontreated macrophage cells as a dosedependent response. The results also showed that, among the tested compounds, RJ and 10-H2DA displayed the highest stimulation of $\mathrm{NO}$ production.

3.8. Effect on the Caspase-3-Like Activity. The caspase-3-like activity of parasites treated with RJ, 10-H2DA, 10-HDAA, and sebacic acid was assessed through the colorimetric protease. The results exhibited that RJ, 10-H2DA, 10HDAA, and sebacic acid significantly induced caspase-3 activation as a dose-dependent response ranging from 9.4 to $27.2 \%$ in comparison with the control (Figure 3). Based on the obtained results, among the tested compounds, 10H2DA and 10-HDAA displayed the maximum induction of caspase-3 activity.

\section{Discussion}

From the last centuries, natural products have been well known as valuable resources of bioactive and useful ingredients for medical, industrial, and agricultural purposes [31]. In recent years, natural products have been evaluated for several procedures in the pest control insecticidal, ovicidal, and evaluation as a repellent [32-34]. The present study aimed to assess the insecticidal, antiplasmodial, antileishmanial, and cytotoxic effects of RJ and its three fatty acids (10-H2DA, 10-HDAA, and sebacic acid). We found that sebacic acid and RJ displayed the lowest and highest larvicidal effects against Ae. aegypti larva, respectively. At present, there is no specific criterion in the recommendations of the WHO for estimating the larvicidal activity of natural products, but several studies have reported some criteria to demonstrate the efficacy of insecticides derived from natural products $[35,36]$. For example, Komalamisra et al. [37] have revealed that natural products with the $\mathrm{LC}_{50}$ value of less than $50 \mathrm{mg} / \mathrm{L}$ are promising and active, while the products with the $\mathrm{LC}_{50}$ values between 50 to $100 \mathrm{mg} / \mathrm{L}$ were moderately active. In addition, natural products with the $\mathrm{LC}_{50}$ value between 100 and $750 \mathrm{mg} / \mathrm{L}$ and $>750 \mathrm{mg} / \mathrm{L}$ were effective and inactive, respectively. Another investigation conducted by Kiran et al. [38] reported that natural products with the $\mathrm{LC}_{50}$ less than $100 \mathrm{mg} / \mathrm{L}$ are considered as a potent larvicidal effect. However, these criteria are depended on some factors such as exposure time and the source of larvae, which can affect the $\mathrm{LC}_{50}$ values of tested natural products [39]. Hence, our results exhibited the relevant and promising insecticidal effects of RJ, 10-H2DA, 
TABLE 1: The results of measurement of the secondary metabolites contents of royal jelly.

\begin{tabular}{lcc}
\hline Total content & Test & Amount \\
\hline Phenolic & Folin-Ciocalteu's reagent colorimetric & $83.6 \pm 0.31 \mathrm{mg}$ GEA/g DW \\
Flavonoids & Aluminum chloride $\left(\mathrm{AlCl}_{3}\right.$ 2\%) colorimetric & $1.78 \pm 0.023 \mathrm{mg} \mathrm{QE} / \mathrm{g} \mathrm{DW}$ \\
Protein & Bradford method & $11.3 \%$ \\
\hline
\end{tabular}

TABLE 2: Larvicidal activity of royal jelly and its three main fatty acids (trans-10-hydroxy-2-decenoic acid (10-H2DA), 10-hydroxydecanoic acid (10-HDAA), and sebacic acid (1,10-decanedioic acid)) against Ae. aegypti larva.

\begin{tabular}{lr}
\hline Drug & Insecticidal activity $\mathrm{LC}_{50}(\mu \mathrm{g} / \mathrm{mL})$ \\
\hline Royal jelly & 24.6 \\
$10-\mathrm{H} 2 \mathrm{DA}$ & 31.4 \\
$10-\mathrm{HDAA}$ & 37.8 \\
Sebacic acid & 44.7 \\
\hline
\end{tabular}

TABLE 3: Antimalarial activity of royal jelly and its three main fatty acids (trans-10-hydroxy-2-decenoic acid (10-H2DA), 10-hydroxydecanoic acid (10-HDAA), and sebacic acid (1,10-decanedioic acid)) against $P$. falciparum K1-strain.

\begin{tabular}{lc}
\hline Drug & Antimalarial activity $\mathrm{IC}_{50}(\mu \mathrm{g} / \mathrm{mL})$ \\
\hline Royal jelly & $7.62 \pm 0.65$ \\
10-H2DA & $2.41 \pm 0.155$ \\
10-HDAA & $2.65 \pm 0.182$ \\
Sebacic acid & $3.1 \pm 0.213$ \\
Chloroquine sulphate & $0.53 \pm 0.213$ \\
\hline
\end{tabular}

Data are presented as the mean \pm SD.

TABLE 4: Antileishmanial activity of royal jelly and its three main fatty acids (trans-10-hydroxy-2-decenoic acid (10-H2DA), 10-hydroxydecanoic acid (10-HDAA), and sebacic acid (1,10-decanedioic acid)) against intracellular amastigotes of Leishmania major.

\begin{tabular}{lc}
\hline Drug & Antileishmanial effect $\mathrm{IC}_{50}(\mu \mathrm{g} / \mathrm{mL})$ \\
\hline Royal jelly & $8.4 \pm 0.74$ \\
10-H2DA & $3.8 \pm 0.22$ \\
10-HDAA & $3.77 \pm 0.46$ \\
Sebacic acid & $4.13 \pm 0.51$ \\
Meglumine antimoniate & $13.6 \pm 1.15$ \\
\hline
\end{tabular}

Data are presented as the mean \pm SD.

TABLE 5: Cytotoxic effects of royal jelly and its three main fatty acids (trans-10-hydroxy-2-decenoic acid (10-H2DA), 10-hydroxydecanoic acid (10-HDAA), and sebacic acid (1,10-decanedioic acid)) and their selectivity index (SI) against human HEK239T normal cells.

\begin{tabular}{lcr}
\hline Drug & Cytotoxicity effect $C_{50}(\mu \mathrm{g} / \mathrm{mL})$ & SI \\
\hline Royal jelly & 117.3 & 13.9 \\
10-H2DA & 74.4 & 19.5 \\
10-HDAA & 81.3 & 21.5 \\
Sebacic acid & 92.6 & 22.4 \\
\hline
\end{tabular}

Data are presented as the mean \pm SD.

10-HDAA, and sebacic acid, according to the criterion reported by Komalamisra et al. [37] and Kiran et al. [38].

Our findings demonstrated that RJ, 10-H2DA, 10HDAA, and sebacic acid had considerable $(P<0.001)$ antiplasmodial effects against $P$. falciparum with the $\mathrm{IC}_{50}$ values $7.62,2.41,2.65$, and $3.1 \mu \mathrm{g} / \mathrm{mL}$, respectively. In addition, we found that RJ, 10-H2DA, 10-HDAA, and sebacic acid had considerable $(P<0.001)$ antileishmanial effects against the intracellular amastigotes of $L$. major with the $\mathrm{IC}_{50}$ value $8.14,3.8,3.77$, and $4.13 \mu \mathrm{g} / \mathrm{mL}$, respectively. Today, there has been rising attention in evaluating the pharmacological and chemical properties of bee-related products as a substitute antiparasitic treatment [40]. Previously, it has been proven that bee-related products such as honey, propolis, bee pollen, and bee venom are widely used as herbal therapies for treating various infectious diseases in various parts of the world [41]. Several investigations have shown the promising in vitro and/or in vivo antiparasitic effects of bee-related products against a wide range of protozoa and helminths such as Schistosoma spp., Trypanosoma spp., Leishmania spp., and Plasmodium spp. [42]. Considering the antimicrobial mechanisms of bee products, previous studies displayed that these compounds have several antimicrobial mechanisms such as (i) disruption of 


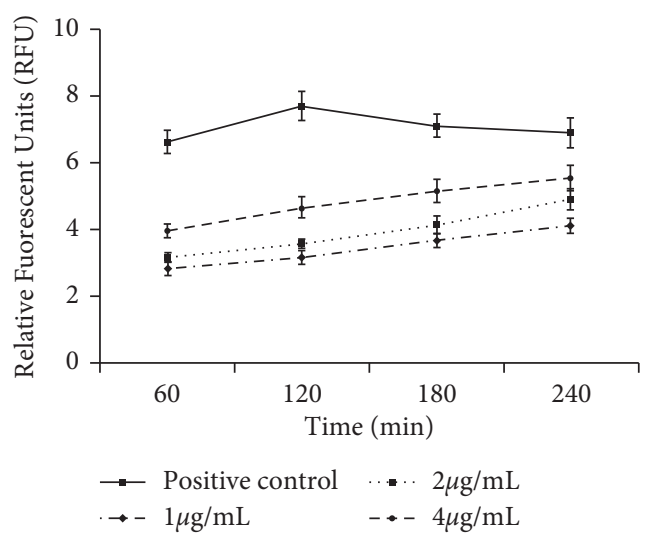

(a)

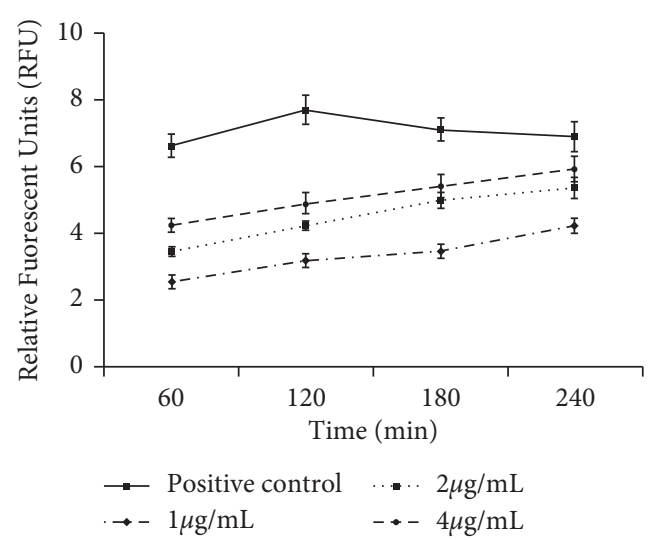

(c)

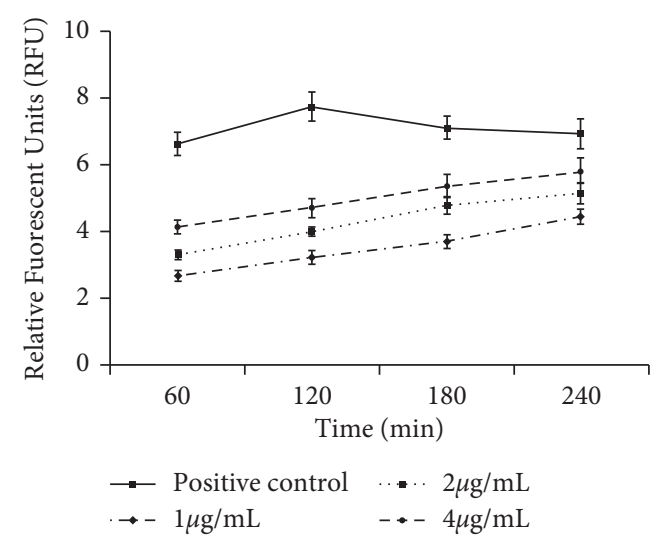

(b)

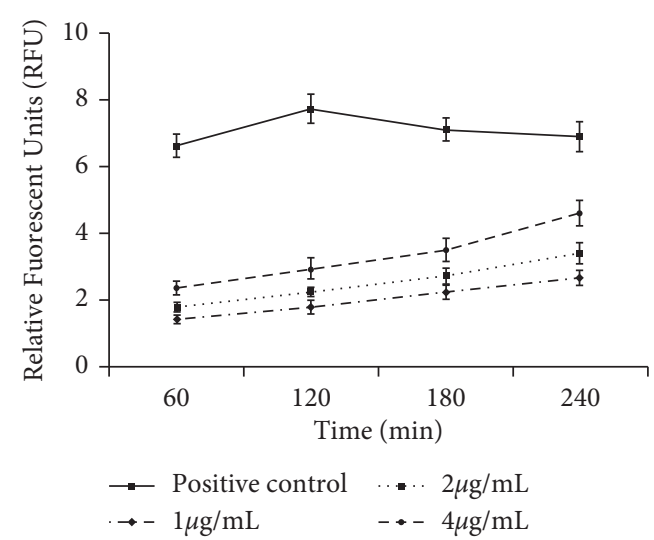

(d)

FIGURE 1: The plasma membrane permeability of the Leishmania major promastigotes treated with RJ (a), 10-H2DA (b), 10-HDAA (c), and sebacic acid (d). The results exhibited of relative fluorescence units revealed that the promastigotes treated with RJ, 10-H2DA, 10-HDAA, and sebacic acid as a dose-dependent response changed the permeability of plasma membrane by Sytox Green. Data are presented as the mean $\pm \mathrm{SD}(n=3)$.

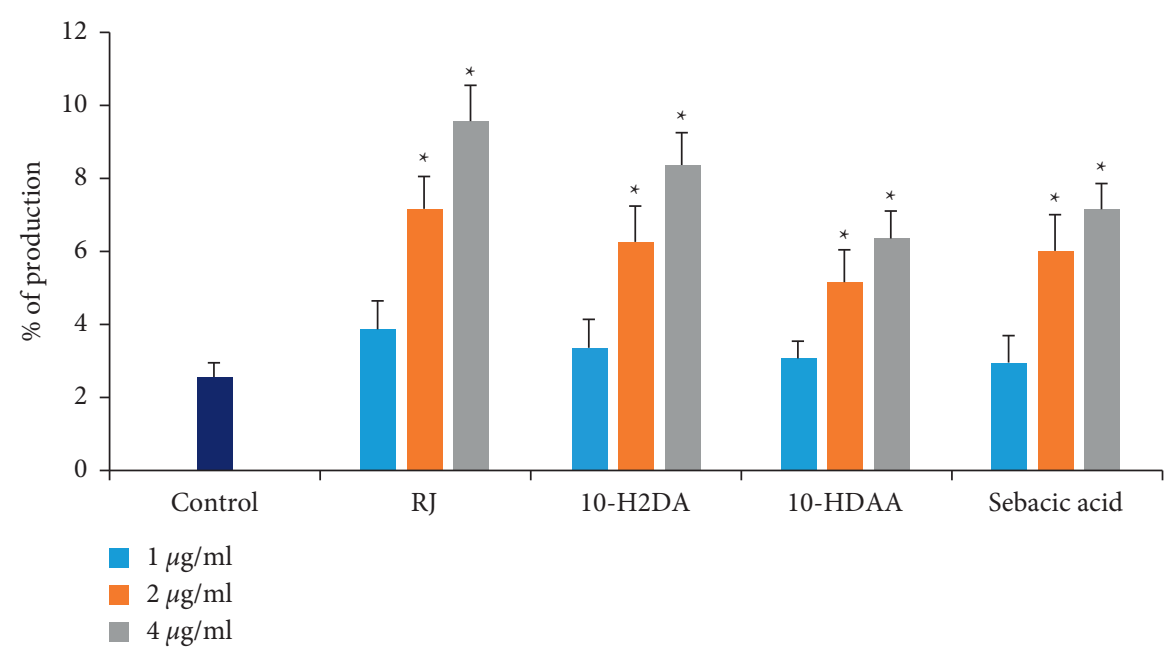

FIGURE 2: Comparison of NO production in J774-A1 macrophage cells after treatment with various concentrations of royal jelly and its three main fatty acids (trans-10-hydroxy-2-decenoic acid (10-H2DA), 10-hydroxydecanoic acid (10-HDAA), and sebacic acid). Data are presented as the mean $\pm \mathrm{SD}(n=3) .{ }^{*} P<0.05$ shows that the difference was statistically significant in comparison with control. 


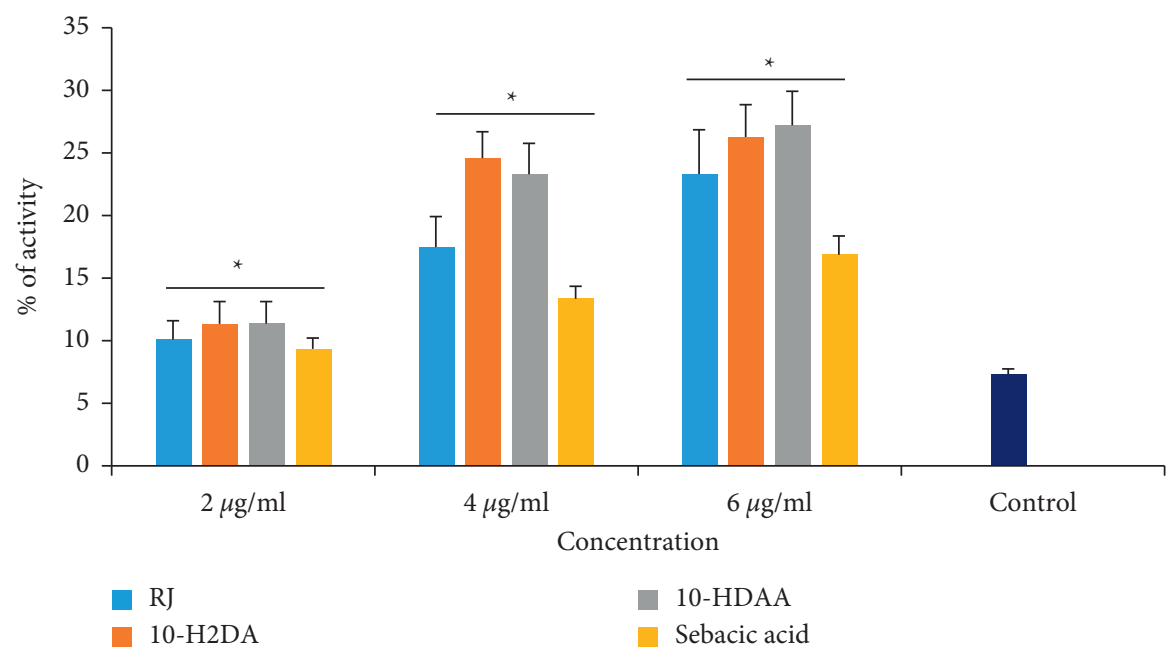

Figure 3: The caspase-3-like activity of promastigotes treated with RJ, 10-H2DA, 10-HDAA, and sebacic acid using the colorimetric protease methods. The results exhibited that RJ, 10-H2DA, 10-HDAA, and sebacic acid significantly induced caspase- 3 activation as a dosedependent response. Data are presented as the mean $\pm \mathrm{SD}(n=3) .{ }^{*} P<0.05$ shows that the difference was statistically significant in comparison with control.

cell wall, (ii) stimulation of macrophages via the production of reactive oxygen species (ROS) and nitrogen metabolites, (iii) promoting the activation of host immune responses by stimulating the production of some cytokines, and (iv) the induction of apoptosis-like mechanisms [43-50].

We found that the10-H2DA, 10-HDAA, and sebacic acid as the major fatty acids present in RJ had potent insecticidal, antileishmanial, and antiplasmodial effects. Reviews have previously demonstrated that fatty acids demonstrated their antimicrobial mechanisms through suppressing the cellular energy production, inhibition of the DNA/RNA replication, inhibition of enzyme activity, nutrient uptake disorders, generation of peroxidation, and autooxidation degradation products, as well as cytoplasmic membrane disruption $[51,52]$. Therefore, it can be suggested that the insecticidal, antiplasmodial, and antileishmanial effects of RJ are due to the existence of these constituents in RJ.

Nowadays, it has been demonstrated that NO, which is produced by a number of immune cells, plays a critical role in the immune-mediated response for eliminating intracellular pathogens [53]. We reported that RJ, 10-H2DA, 10HDAA, and sebacic acid $(1,2$, and $4 \mu \mathrm{g} / \mathrm{mL})$ significantly $(P<0.05)$ increased the production of $\mathrm{NO}$ as a dose-dependent pattern in comparison to the nontreated macrophage cells. These results suggest that although RJ, 10H2DA, 10-HDAA, and sebacic acid triggered the NO production as an important intracellular antimicrobial mechanism, supplementary surveys and analyses are required to evaluate the importance of $\mathrm{NO}$ and eliminate other factors.

Previous studies have exhibited that the rupture plasma membrane is one of the key action modes to inhibit the growth of intracellular pathogens $[31,40]$. We found that the promastigotes treated with RJ, 10-H2DA, 10-HDAA, and sebacic acid changed the permeability of the plasma membrane by Sytox Green as a dose-dependent response.

Apoptosis is one of the important processes that basically links pathogen survival to its ability to induce controlled death [43]. Among the caspases, as the main mediators of apoptosis, caspase- 3 is considered one of the key caspases that predominantly triggered death protease and successively prompt cell death [45]. Here, we found that RJ, 10H2DA, 10-HDAA, and sebacic acid significantly induced caspase- 3 activation as a dose-dependent response ranging from 9.4 to $27.2 \%$ in comparison with the control. Considering the cytotoxicity effects of RJ, 10-H2DA, 10-HDAA, and sebacic acid, the obtained results of the MTT assay demonstrated that the $\mathrm{CC}_{50}$ value for RJ, 10-H2DA, 10HDAA, and sebacic acid was 117.3, 74.4, 81.3, and 92.6, respectively; subsequently, their SI $>10$ of these compound exhibited their specificity to parasites and safety against human HEK239T normal cells.

\section{Conclusion}

The results of the present investigation revealed the promising insecticidal, antiplasmodial, and antileishmanial effects of RJ and its three main fatty acids (10-H2DA, 10HDAA, and sebacic acid) against healthy 4th instar larvae of Ae. aegypti, chloroquine-resistant $P$. falciparum K1-strain, and L. major amastigotes, respectively. Although the main mechanisms of action in these natural products are clearly understood, our study revealed that RJ, 10-H2DA, 10HDAA, and sebacic acid displayed their antimicrobial mechanism through the plasma membrane permeability, triggering the NO production, and the induction of apoptosis. More studies are required to confirm the efficacy of these compounds, especially in animal models and clinical settings. 


\section{Data Availability}

All data generated or analyzed during this study are included in this published paper.

\section{Consent}

Not applicable.

\section{Conflicts of Interest}

All authors declare that they have no conflicts of interest.

\section{Acknowledgments}

The authors thank the staff members of the Biological Science Department, Faculty of Science and Humanities, Shaqra University, and the staff members of the Department of Biology, Faculty of Science, University of Tabuk, Saudi Arabia, for their technical support.

\section{References}

[1] WHO, "Vector-borne diseases. Fact sheets 2017," 2019, https://www.who.int/news-room/factsheets/detail/vectorborne-diseases.

[2] J. A. Souza-Neto, J. R. Powell, and M. Bonizzoni, "Aedes aegypti vector competence studies: a review," Infection, Genetics and Evolution, vol. 67, pp. 191-209, 2019.

[3] J. R. Powell and W. J. Tabachnick, "History of domestication and spread of Aedes aegypti-a review," Memórias do Instituto Oswaldo Cruz, vol. 108, no. suppl 1, pp. 11-17, 2013.

[4] N. Sutthanont, S. Attrapadung, and S. Nuchprayoon, "Larvicidal activity of synthesized silver nanoparticles from Curcuma zedoaria essential oil against Culex quinquefasciatus," Insects, vol. 10, no. 1, p. 27, 2019.

[5] T. Chareonviriyaphap, M. J. Bangs, W. Suwonkerd, M. Kongmee, V. Corbel, and R. Ngoen-Klan, "Review of insecticide resistance and behavioral avoidance of vectors of human diseases in Thailand," Parasites \& Vectors, vol. 6, no. 1, p. 280, 2013.

[6] R. Pavela, "Larvicidal effects of various Euro-Asiatic plants against Culex quinquefasciatus Say larvae (Diptera: Culicidae) Parasitol," Parasitology Research, vol. 102, no. 3, pp. 555-559, 2008.

[7] A. Abdul Rahuman, G. Gopalakrishnan, P. Venkatesan, and K. Geetha, "Isolation and identification of mosquito larvicidal compound from Abutilon indicum (Linn.)," Parasitology Research, vol. 102, no. 5, pp. 981-988, 2008.

[8] M. Bannister-Tyrrell, K. Verdonck, S. Hausmann-Muela, C. Gryseels, J. M. Ribera, and K. P. Grietens, "Defining microepidemiology for malaria elimination: systematic review and meta-analysis," Malaria Journal, vol. 16, no. 1, pp. 1-20, 2017.

[9] K. Sabina, "Prevalence and epidemiology of malaria in Nigeria: a review," International Journal of Research in Pharmacy and Biosciences, vol. 4, no. 8, pp. 10-12, 2017.

[10] R. E. Cibulskis, P. Alonso, J. Aponte et al., "Malaria: global progress 2000-2015 and future challenges," Infectious diseases of poverty, vol. 5, no. 1, pp. 1-8, 2016.

[11] G. Newby, J. Hwang, K. Koita et al., "Review of mass drug administration for malaria and its operational challenges," The American Journal of Tropical Medicine and Hygiene, vol. 93, no. 1, pp. 125-134, 2015.
[12] I. Kevric, M. A. Cappel, and J. H. Keeling, "New world and old world Leishmania infections: a practical review," Dermatologic Clinics, vol. 33, no. 3, pp. 579-593, 2015.

[13] I. Sharifi, M. R. Aflatoonian, M. H. Daei Parizi et al., "Visceral leishmaniasis in Southeastern Iran: a narrative review," Iranian Journal of Parasitology, vol. 12, no. 1, pp. 1-11, 2017.

[14] L. Monzote, "Current treatment of leishmaniasis: a review," The Open Antimicrobial Agents Journal, vol. 1, no. 1, 2009.

[15] L. F. Oliveira, A. O. Schubach, M. M. Martins et al., "Systematic review of the adverse effects of cutaneous leishmaniasis treatment in the New World," Acta Tropica, vol. 118, no. 2, pp. 87-96, 2011.

[16] G. M. Cragg and D. J. Newman, "Natural products: a continuing source of novel drug leads," Biochimica et Biophysica Acta (BBA)-General Subjects, vol. 1830, no. 6, pp. 3670-3695, 2013.

[17] V. R. Pasupuleti, L. Sammugam, N. Ramesh, and S. H. Gan, "Honey, propolis, and royal jelly: a comprehensive review of their biological actions and health benefits," Oxidative Medicine and Cellular Longevity, vol. 2017, Article ID 1259510, 2017.

[18] C. I. Pavel, L. A. Mărghitaş, O. Bobiş et al., "Biological activities of royal jelly-review," Scientific Papers Animal Science and Biotechnologies, vol. 44, no. 2, pp. 108-118, 2011.

[19] M. F. Ramadan and A. Al-Ghamdi, "Bioactive compounds and health-promoting properties of royal jelly: a review," Journal of Functional Foods, vol. 4, no. 1, pp. 39-52, 2012.

[20] J. Kocot, M. Kiełczykowska, D. Luchowska-Kocot, J. Kurzepa, and I. Musik, "Antioxidant potential of propolis, bee pollen, and royal jelly: possible medical application," Oxidative Medicine and Cellular Longevity, vol. 2018, Article ID 7074209, 2018.

[21] S. Ahmad, M. G. Campos, F. Fratini, S. Z. Altaye, and J. Li, "New insights into the biological and pharmaceutical properties of royal jelly," International Journal of Molecular Sciences, vol. 21, no. 2, p. 382, 2020.

[22] S. Kolayli, H. Sahin, Z. Can, O. Yildiz, M. Malkoc, and A. Asadov, "A member of complementary medicinal food: anatolian royal jellies, their chemical compositions, and antioxidant properties. J. Evid. Based complement," Alternative Medicine, vol. 21, pp. Np43-NP48, 2016.

[23] V. A. Isidorov, S. Bakier, and I. Grzech, "Gas chromatographic-mass spectrometric investigation of volatile and extractable compounds of crude royal jelly," Journal of Chromatography B, vol. 885-886, pp. 109-116, 2012.

[24] E. Melliou and I. Chinou, "Chemistry and bioactivity of royal jelly from Greece," Journal of Agricultural and Food Chemistry, vol. 53, no. 23, pp. 8987-8992, 2005.

[25] V. L. Singleton, R. Orthofer, and R. M. Lamuela-Raventós, "Analysis of total phenols and other oxidation substrates and antioxidants by means of Folin-Ciocalteu reagent," Oxidants and Antioxidants Part A, vol. 299, pp. 152-178, 1999.

[26] S. El-Guendouz, S. Aazza, B. Lyoussi, M. D. Antunes, M. L. Faleiro, and M. G. Miguel, "Anti-acetylcholinesterase, antidiabetic, anti-inflammatory, antityrosinase and antixanthine oxidase activities of Moroccan propolis," International Journal of Food Science and Technology, vol. 51, no. 8, pp. 1762-1773, 2016.

[27] K. Hartfelder, M. M. G. Bitondi, C. S. Brent et al., "Standard methods for physiology and biochemistry research inApis mellifera," Journal of Apicultural Research, vol. 52, no. 1, pp. 1-48, 2013.

[28] L. T. Huong, N. H. Hung, D. N. Dai et al., "Chemical compositions and mosquito larvicidal activities of essential 
oils from Piper species growing wild in Central Vietnam," Molecules, vol. 24, no. 21, p. 3871, 2019.

[29] M. T. Makler, R. C. Piper, J. A. Williams et al., "Parasite lactate dehydrogenase as an assay for Plasmodium falciparum drug sensitivity," The American Journal of Tropical Medicine and Hygiene, vol. 48, no. 6, pp. 739-741, 1993.

[30] A. E. Albalawi, S. Abdel-Shafy, A. Khudair Khalaf et al., "Therapeutic potential of green synthesized copper nanoparticles alone or combined with meglumineantimoniate (glucantime ${ }^{\circledR}$ ) in cutaneous leishmaniasis," Nanomaterials, vol. 11, no. 4, p. 891, 2021.

[31] H. Yuan, Q. Ma, L. Ye, and G. Piao, "The traditional medicine and modern medicine from natural products," Molecules, vol. 21 , no. 5, p. 559, 2016.

[32] A. Asadollahi, M. Khoobdel, A. Zahraei-Ramazani, S. Azarmi, and S. H. Mosawi, "Effectiveness of plant-based repellents against different Anopheles species: a systematic review," Malaria Journal, vol. 18, no. 1, pp. 436-520, 2019.

[33] M. B. Isman, "Botanical insecticides, deterrents, and repellents in modern agriculture and an increasingly regulated world," Annual Review of Entomology, vol. 51, no. 1, pp. 45-66, 2006.

[34] A. Ghosh, N. Chowdhury, and G. Chandra, "Plant extracts as potential mosquito larvicides," Indian Journal of Medical Research, vol. 135, no. 5, pp. 581-98, 2012.

[35] J.-M. Chantraine, D. Laurent, C. Ballivian, G. Saavedra, R. Ibañez, and L. A. Vilaseca, "Insecticidal activity of essential oils on Aedes aegypti larvae," Phytotherapy Research, vol. 12, no. 5, pp. 350-354, 1998.

[36] L. A. Magalhães, M. P. Lima, M. O. Marques, R. Facanali, A. C. Pinto, and W. P. Tadei, "Chemical composition and larvicidal activity against Aedes aegypti larvae of essential oils from four Guarea species," Molecules, vol. 15, no. 8, pp. 5734-5741, 2010.

[37] N. Komalamisra, Y. Trongtokit, Y. Rongsriyam, and C. Apiwathnasorn, "Screening for larvicidal activity in some Thai plants against four mosquito vector species," The Southeast Asian journal of tropical medicine and public health, vol. 36, no. 6, pp. 1412-22, 2005.

[38] S. Ravi Kiran, K. Bhavani, P. Sita Devi, B. R. Rajeswara Rao, and K. Janardhan Reddy, "Composition and larvicidal activity of leaves and stem essential oils of Chloroxylon swietenia DC against Aedes aegypti and Anopheles stephensi," Bioresource Technology, vol. 97, no. 18, pp. 2481-2484, 2006.

[39] C. N. Dias, L. P. Alves, K. A. Rodrigues et al., "Chemical composition and larvicidal activity of essential oils extracted from Brazilian legal Amazon plants against Aedes aegypti L.(Diptera: Culicidae)," Evidence-based Complementary and Alternative Medicine, vol. 2015, Article ID 490765, 2015.

[40] R. Cauich-Kumul and M. R. Segura Campos, "Bee propolis: properties, chemical composition, applications, and potential health effects," Bioactive Compounds, Woodhead Publishing, vol. 12, pp. 227-243, 2019.

[41] S. El-Guendouz, B. Lyoussi, and M. G. Miguel, "Insight on propolis from mediterranean countries: chemical composition, biological activities and application fields," Chemistry and Biodiversity, vol. 16, no. 7, Article ID e1900094, 2019.

[42] F. Nainu, A. Masyita, M. A. Bahar et al., "Pharmaceutical prospects of bee products: special focus on anticancer, antibacterial, antiviral, and antiparasitic properties," Antibiotics, vol. 10, no. 7, p. 822, 2021.

[43] R. P. Dutra, J. L. Bezerra, M. C. P. d. Silva et al., "Antileishmanial activity and chemical composition from Brazilian geopropolis produced by stingless bee Melipona fasciculata,"
Revista Brasileira de Farmacognosia, vol. 29, no. 3, pp. 287293, 2019.

[44] A. N. P. Gomes, C. A. Camara, A. dos Santos Sousa et al., "Chemical composition of bee pollen and leishmanicidal activity of rhusflavone," Revista Brasileira de Farmacognosia, vol. 31, no. 2, pp. 176-183, 2021.

[45] O. Cuesta-Rubio, M. Campo Fernández, I. Márquez Hernández et al., "Chemical profile and anti-leishmanial activity of three Ecuadorian propolis samples from Quito, Guayaquil and Cotacachi regions," Fitoterapia, vol. 120, pp. 177-183, 2017.

[46] B. C. Cunha, M. B. De Miranda, L. C. C. Afonso et al., "Brazilian green propolis hydroalcoholic extract as a therapeutic adjuvant to treat cutaneous leishmaniasis," Journal of Applied Pharmaceutical Science, vol. 10, pp. 124-132, 2020.

[47] A. P. F. dos Santos Thomazelli, F. Tomiotto-Pellissier, S. S. da Silva et al., "Brazilian propolis promotes immunomodulation on human cells from American Tegumentar Leishmaniasis patients and healthy donors infected with L. braziliensis," Cellular Immunology, vol. 311, pp. 22-27, 2017.

[48] S. Yesmin, A. Paul, T. Naz et al., "Membrane stabilization as a mechanism of the anti-inflammatory activity of ethanolic root extract of Choi (Piper chaba)," Clinical Phytoscience, vol. 6, no. 1, p. 59, 2020.

[49] A. G. Hegazi, F. M. Al Guthami, A. F. Al Gethami, and H. A. El Fadaly, "Beneficial effects of Capparis spinosa honey on the immune response of rats infected with toxoplasma gondii," Journal of Pharmacopuncture, vol. 20, pp. 112-118, 2017.

[50] R. F. S. Menna-Barreto, K. Salomão, A. P. Dantas et al., "Different cell death pathways induced by drugs in Trypanosoma cruzi: an ultrastructural study," Micron, vol. 40, no. 2, pp. 157-168, 2009.

[51] A. P. Desbois and V. J. Smith, "Antibacterial free fatty acids: activities, mechanisms of action and biotechnological potential," Applied Microbiology and Biotechnology, vol. 85, no. 6, pp. 1629-1642, 2010.

[52] G. Casillas-Vargas, C. Ocasio-Malavé, S. Medina et al., "Antibacterial fatty acids: an update of possible mechanisms of action and implications in the development of the nextgeneration of antibacterial agents," Progress in Lipid Research, vol. 82, Article ID 101093, 2021.

[53] P. Holzmuller, D. Sereno, M. Cavaleyra et al., "Nitric oxidemediated proteasome-dependent oligonucleosomal DNA fragmentation in Leishmaniaamazonensis amastigotes," Infection and Immunity, vol. 70, no. 7, pp. 3727-3735, 2002. 